\title{
Análise dos investimentos em sistemas de aproveitamento de água pluvial nas cidades do sertão Alagoano
}

\author{
CORREIA, Lucas Miguel Alencar de Morais ${ }^{1}$ \\ SOUZA, Beatriz Pereira de ${ }^{2}$ \\ COELHO, Everton de Souza ${ }^{3}$ \\ SILVA, Maria Katarina Gomes da ${ }^{4}$ \\ LIMA, Stefany Gonçalves ${ }^{5}$ \\ PEREIRA, Thiago Alberto da Silva ${ }^{6}$ \\ 1Universidade Federal de Alagoas, Delmiro Gouveia, Brasil, lucasalencar1000@hotmail.com \\ 2Universidade Federal de Alagoas, Delmiro Gouveia, Brasil, pereirabeat97@gmail.com \\ ${ }^{3}$ Universidade Federal de Alagoas, Delmiro Gouveia, Brasil, everton.coelho@delmiro.ufal.br \\ 4Universidade Federal de Alagoas, Delmiro Gouveia, Brasil, katari-naga@hotmail.com \\ 5Universidade Federal de Alagoas, Delmiro Gouveia, Brasil, stefglima@gmail.com \\ 6Universidade Federal de Alagoas, Delmiro Gouveia, Brasil, Thiago_alb@hotmail.com
}

\begin{abstract}
Resumo
A escassez dos recursos hídricos impulsiona a busca por técnicas inovadoras capazes de motivar o aproveitamento de águas pluviais, viabilizando a economia de água potável e reduzindo a escassez, simultaneamente. Diante disso, o presente estudo busca estimar o valor necessário investido em três municípios do sertão alagoano em sistemas de aproveitamento de água pluvial. Para tal, foi mensurada neste trabalho a capacidade de aproveitamento de água de chuva para três municípios que estão localizados no extremo oeste de Alagoas, após isso, foi orçado o sistema necessário para condução de água da chuva e por fim, foi analisado o investimento à luz do Plano Plurianual do estado de Alagoas. Resultados apontam que as cidades possuem potencial de aproveitamento de água pluvial acima de $50 \%$, na esfera orçamentária encontrou-se um investimento na ordem 4,6 milhões de reais que quando confrontando com as políticas públicas, mostrou-se que este investimento representa menos de $1 \%$ do que foi previsto para Plano Plurianual do estado, voltado para obras e alternativas de abastecimento de água. A efetivação desta política trará benefícios nas diversas esferas da sociedade, como geração de renda, saúde pública e crescimento econômico.
\end{abstract}

Palavras-Chave: Aproveitamento de águas pluviais; Economia; Políticas Públicas.

\begin{abstract}
The shortage of water resources drives the search by means of a water economy and a scarcity simultaneously. In view of this, the first study needs to be able to invest in three municipalities of the Alagoas system in systems for the utilization of rainwater. To do so, the measures for the abandonment of the level of water for the rains are located in no extreme west of Alagoas, after that, the system for the measurement of rain was budgeted and, finally, the State's Pluriannual Plan Light investment was analyzed of Alagoas. Results that have a historical potential for the use of rainwater over $50 \%$ in the budget architecture, with an investment of around 4.6 million reais when compared to public forecasts, this investment represents less than $1 \%$ of what was foreseen for the Plurianual Plan of the state, directed to works and alternatives of water supply. The implementation of this policy has benefits in various spheres of society, such as income generation, public health and economic growth.
\end{abstract}

Key-Words: Utilization of Rainwater; Economy; Public Forecasts. 


\section{Introdução}

A água é a fonte de vida de toda espécie do planeta Terra. Trata-se de um componente indispensável para a perpetuação da humanidade. Sendo o único recurso natural que está presente em todos os aspectos da vida humana, sejam eles sociais econômicos ou religiosos, deve-se pensar em alternativas para o melhor aproveitamento e funcionamento desse recurso na sociedade.

O Brasil é um país privilegiado no que diz respeito à quantidade de água. Possui inúmeras reservas naturais que são consideradas uma das maiores do mundo, todavia a distribuição desse recurso ocorre de maneira não uniforme de acordo com a localização geográfica (PENA, 2016). Quando se trata da região Nordeste, onde as secas sempre constituíram desastres sociais e econômicos, desde os séculos passados a situação passa por diversos agravantes que comprometem diretamente o funcionamento da vida familiar.

O aproveitamento das águas pluviais incide como uma medida que possibilita a redução na escassez de água, presente, sobretudo na região Nordeste (ANDRADE, 2015). A utilização dessa técnica envolve a implantação de elementos apropriados, visando à captação, condução e armazenamento adequados, envolvendo fatores como eliminação de desperdícios e qualidade da água coletada, além de apresentar a importância das políticas públicas para viabilizar o sistema. Diante disso, o presente estudo visa orçar o montante de investimento necessário para implantação dos elementos de condução de água pluviais em cidades que são constantemente castigadas pelo desabastecimento dos sistemas de distribuição de água tratada e analisar o montante, desse investimento, à luz das políticas públicas.

\section{Metodologia}

\section{1. Área de Estudo}

O Sistema Coletivo do Sertão Alagoano é responsável pelo abastecimento de sete municípios da mesorregião do sertão do estado, sendo eles: Água Branca, Canapi, Delmiro Gouveia, Inhapi, Mata Grande, Olho d'Água do Casado e Pariconha, localizadas no extremo oeste do estado e com, aproximadamente, 150 mil habitantes. Entretanto, devido à ausência de continuidade referente à distribuição, alguns desses municípios são escanteados pelo regime de abastecimento, onde os casos mais consideráveis foram os das cidades de Canapi, Inhapi e Mata Grande, por localizarem-se no limite da zona de abastecimento e por possuir localização geográfica desprivilegiada. Com isso, foi identificado o desabastecimento constante nessas localidades, tendo apenas um rodízio na distribuição de água, que ultrapassam mais de 30 dias seguidos.

Por conta disso, a população destas cidades (Canapi, Inhapi e Mata Grande) construiu cisternas nas suas residências para acumulação de água fornecida pela concessionária e utilizar para os dias que não há abastecimento, além disso, a depender do sistema de rodízio, os entes governamentais também utilizam carros-pipa para mitigar os efeitos do desabastecimento de água tratada, portanto, objeto deste trabalho será essas três cidades.

\subsection{Cálculo do Potencial de Aproveitamento de Água Pluvial}

Antes de levantar os investimentos necessários para condução de água de chuva, verificou-se o potencial de economia de água potável, a partir da coleta de águas pluviais através da metodologia apresentada por Ghisi et al. (2006). A seguir as equações utilizadas para aplicação desta metodologia. 
Tabela 1: Equações Utilizadas

\begin{tabular}{|c|c|c|}
\hline Equação & & Variáveis \\
\hline$P D=\frac{P C}{N D C}$ & $(1)$ & $\begin{array}{l}\text { PC: Total populacional de cada cidade } \\
\text { NDC: Quantidade de ligações ativas de água em cada } \\
\text { cidade. PD: Pessoas por domicílio. }\end{array}$ \\
\hline$N D=\frac{\mathrm{NP}}{P D}$ & $(2)$ & $\begin{array}{l}\text { NP: Total de pessoas atendidas pelo abastecimento de } \\
\text { água em cada cidade. }\end{array}$ \\
\hline$R A=H \times 85+F \times P D \times 3,75$ & $(3)$ & $\begin{array}{l}\text { H: Porcentagem de casas } \\
\text { F: Porcentagem de apartamentos } \\
\text { RA: Área média do telhado }\end{array}$ \\
\hline$T R A=R A \times N D$ & $(4)$ & TRA: Área total de telhado \\
\hline$V R=\frac{R \times T R A \times R c}{1000}$ & $(5)$ & $\begin{array}{l}\text { R: Dados mensais de precipitação; } \\
\text { Rc: Coeficiente de Runoff. } \\
\text { VR: Volume de Chuva }\end{array}$ \\
\hline$P P W S_{m}=100 \frac{V R}{P W R}$ & $(6)$ & $\begin{array}{l}\text { PWR: Consumo médio mensal de água do sistema. } \\
\text { PPWS }_{\mathrm{m}} \text { : Potencial mensal de economia de água }\end{array}$ \\
\hline$P P W S=\frac{\sum P P W S_{i} \times V R_{i}}{\sum V R_{i}}$ & $(7)$ & PPWS: Potencial anual de economia \\
\hline
\end{tabular}

Fonte: Ghisi et. al (2006)

\subsection{Análise Econômica}

Para o custo de implantação do sistema de água pluvial nas cidades, assumimos que nas residências existem reservatórios para o armazenamento da água captada, conforme descrito no objeto de estudo. Diante disso, apresentaremos o custo de implantação do sistema e os dispositivos de descartes da primeira água, preservando a boa qualidade da mesma nas cidades de Inhapi, Canapi e Mata Grande.

Inicialmente, para o direcionamento do cálculo das calhas, utilizaremos a área do telhado estimada. Pelo método de Ghisi et al. (2006) é estimado uma área de $85 \mathrm{~m}^{2}$ para casas, no qual foi definido como um retângulo de $8,5 \mathrm{~m}$ de frente e 10,0 m de comprimento. Para o cálculo da quantidade de metros de calha de cada município (MC) é utilizada a equação 9.

$$
M C=17 \times N D
$$

Para os condutores (elemento que leva água da calha para as cisternas), em cada residência foi adotado que seriam necessários 12,5 metros de cano PVC de $100 \mathrm{~mm}$. O cálculo do quantitativo de condutores (CC) para cada município é dado pela equação 10.

$$
C C=12,5 \times N D
$$

Para o cálculo do equipamento que será utilizado para o descarte das primeiras águas, visto que pela NBR (15527/07) recomenda-se o descarte dos dois primeiros milímetros. Diante disso, o equipamento necessário utilizará 22 metros de cano PVC com o diâmetro de $100 \mathrm{~mm}$. Logo, para cada cidade, o quantitativo de tubos para descarte (CD) é dado pela equação 11.

$$
C D=22 \times N D
$$

Na realização do cálculo do custo de implantação do sistema de coleta, utilizaremos o menor preço encontrado no mercado, tanto para calhas como para cano PVC, que será multiplicada a quantidade de metros necessários por cidade, e no final será somado todo o investimento para os três municípios.

\section{Resultados e Discussões}

\subsection{Coleta de Dados}

Das 3 cidades referentes à pesquisa, 2 delas apresentaram dados disponíveis de precipitação, entretanto, o município de Inhapi não possui dados em relação à pluviosidade média mensal, deste 
modo foi utilizado o valor de Canapi para a mesma. Essa escolha foi feita, devido as semelhanças geográficas e por serem cidades circunvizinhas. A Figura 01 traz os índices pluviométricos mensais dos municípios estudados.

Figura 1: Precipitação Mensal dos Municípios Estudados.

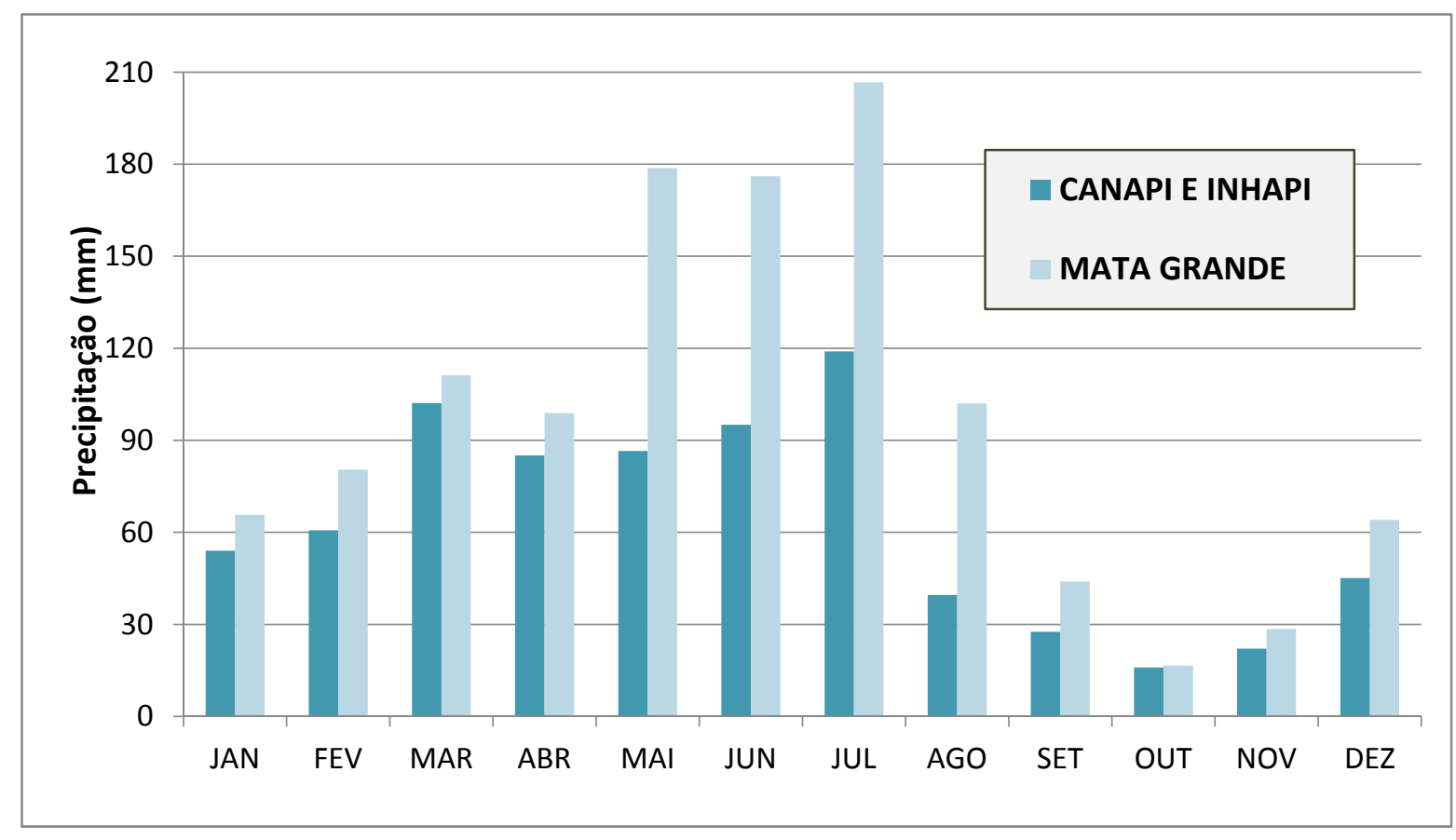

Fonte: Autores (2018)

Os índices pluviométricos anuais das cidades estudadas são de 1172,9 mm e 752,76 mm, respectivamente para a cidade de Mata Grande e Canapi. Valores esses maiores que os encontrados no semiárido, que modo geral, giram em torno de $600 \mathrm{~mm}$, isto pode ser explicado pelo fato das cidades estarem situadas em grandes atitudes que ocasionam clima mais amenos e chuvas orográficas. Esses maiores volumes de chuva podem justificar a utilização de políticas voltadas ao incentivo de aproveitamento de água pluvial.

As informações coletadas pelo Sistema Nacional de Informações de Saneamento (SNIS) estão dispostas na Tabela 02, as quais são: o montante de habitantes por município, o número de ligações ativas de água em cada cidade e o consumo médio mensal de água. De acordo com a Tabela 02, observa-se que as três cidades possuem, consumos semelhantes, mesmo com as diferenças referentes aos dados de população e número de ligações. A cidade de Mata Grande possui o maior índice de consumo médio mensal de água, devido ao maior número de habitantes.

Tabela 2: População, Ligações Ativas e Consumo Médio Mensal de Água.

\begin{tabular}{lccc}
\hline Município & Canapi & Inhapi & Mata Grande \\
\hline População (PC) - hab. & 5483 & 8347 & 9836 \\
\hline Ligações Ativas (NDC) & 1670 & 2113 & 2216 \\
\hline Consumo médio mensal de água (PWR) $-m^{3}$ & $16.191,67$ & $17.764,17$ & $17.974,17$ \\
\hline \multicolumn{4}{c}{ Fonte: SNIS (2016) }
\end{tabular}

\subsection{Potencial de Economia de Água Potável}

A Tabela 03 traz os resultados oriundos da aplicação do método proposto por Ghisi et al (2006). De acordo com os valores estão apresentados na Tabela 03, observa-se que o número de habitante por domicilio gira em torno de 4 pessoas. Admitindo-se que estas cidades não possuem apartamento, a área total de telhados referente às habitações dos municípios estudados ultrapassa o valor de 140 mil 
$\mathrm{m}^{2}$, sendo a menor área pertence à cidade de Canapi e a maior área à Mata Grande. Ainda na Tabela 03, observa-se que a cidade de Mata Grande possui maior potencial de captação por possuir maiores índices pluviométricos e maior população entre as cidades estudadas. No entanto, as três cidades apresentam alto potencial de economia anual, sempre superior a $50 \%$, com destaque para Mata Grande com 83,95\%.

Tabela 3: Cálculo do Potencial de Aproveitamento de Água Pluvial.

\begin{tabular}{|c|c|c|c|}
\hline Município & Canapi & Inhapi & Mata Grande \\
\hline Pessoas por Domicilio (PD) & 3,28 & 3,95 & 4,44 \\
\hline Domicílios Abastecidos (ND) & 1670 & 2113 & 2216 \\
\hline Área do Telhado (TRA) $m^{2}$ & 141.950 & 179.605 & 188.360 \\
\hline Volume de Chuva Coletado $\left(10^{4} \mathrm{~m}^{3}\right)$ & 8,55 & 10,82 & 17,67 \\
\hline Potencial de Economia Anual (\%) & 55,95 & 64,52 & 83,95 \\
\hline
\end{tabular}

De acordo com os cálculos realizados, foi possível determinar o potencial de economia de água potável anual (PPWS), que estão na Tabela 03, em se tratando da análise mensal os valores podem chegar a $100 \%$ de aproveitamento com água pluvial, sobretudo na cidade de Mata Grande. A Figura 2, por sua vez traz os valores anuais aproveitáveis e não aproveitáveis.

Figura 2: Volume Aproveitável e Não Aproveitável Anual.

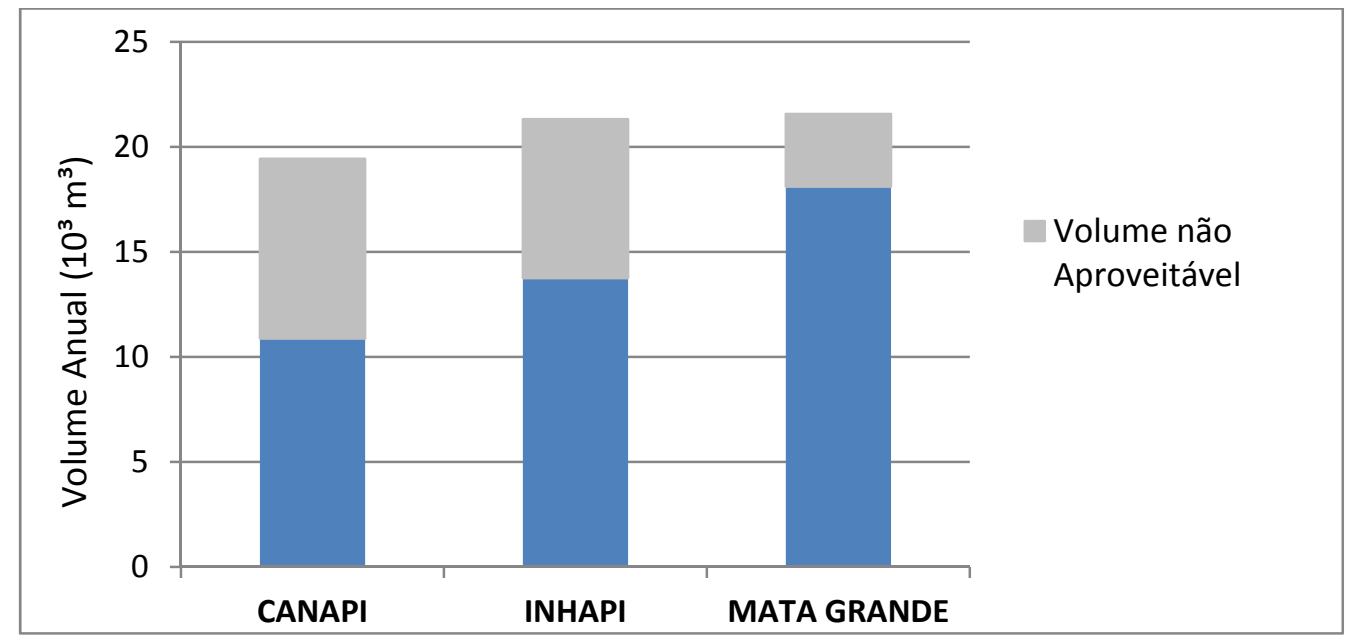

Fonte: Autores (2018)

\subsection{Levantamento do investimento para implantação de calhas}

$\mathrm{Na}$ Tabela 04 pode-se verificar o custo para implantação do sistema de condução de água pluvial nas residências das três cidades estudadas, os valores foram obtidos através de pesquisas de preços para materiais utilizados.

Tabela 4: Custo para Implantação do Sistema de Condução

\begin{tabular}{lccc}
\hline Municípios & Canapi & Mata Grande & Inhapi \\
\hline $\mathrm{ND}$ & 1670 & 2113 & 2216 \\
\hline $\mathrm{MC}$ (Metros) & 28390 & 48866,5 & 32698,65 \\
\hline $\mathrm{CC}$ (Metros) & 20875 & 26412,5 & 27700 \\
\hline $\mathrm{CD}$ (Metros) & 36740 & 62218,5525 & 41633,07525 \\
\hline$\$ M C$ & $\mathrm{R} \$ 823.310,00$ & $\mathrm{R} \$ 1.417 .128,50$ & $\mathrm{R} \$ 948.260,85$ \\
\hline$\$ C C$ & $\mathrm{R} \$ 137.775,00$ & $\mathrm{R} \$ 174.322,50$ & $\mathrm{R} \$ 182.820,00$ \\
\hline$\$ C D$ & $\mathrm{R} \$ 242.484,00$ & $\mathrm{R} \$ 410.642,45$ & $\mathrm{R} \$ 274.778,30$ \\
\hline Investimento & $\mathrm{R} \$ 1.203 .569,00$ & $\mathrm{R} \$ 2.002 .093,45$ & $\mathrm{R} \$ 1.405 .859,15$ \\
\hline Investimento Total & & $\mathrm{R} \$ 4.611 .521,59$ \\
\hline
\end{tabular}


Nota: ND - Número de Domicílios; MC - Quantitativo de Calhas; CC - Quantitativo de Condutores; CD Quantitativo no Sistema de Descarte; \$MC - Investimento em Calhas; CC - Investimento em Condutores; CD Investimento no Sistema de Descarte.

$$
\text { Fonte: Autores (2018) }
$$

Analisando-se a Tabela 04, estimou-se um investimento no valor de $\mathrm{R} \$ 4.611 .521,59$; se for analisado por domicilio verifica-se um valor per capita $\mathrm{R} \$ 768,72$; um valor que pode ser considerado alto, portanto a necessidade de subsidio de por parte dos entes governamentais no intuito de incentivar a instalação deste sistema.

\subsection{O Papel e importância da política pública para viabilizar o aproveitamento da água pluvial}

Vasconcellos e Garcia (2014) expõem que um governo deve perseguir as metas de alto nível de emprego, estabilidade de preços, crescimento econômico e distribuição equitativa da renda, quando estabelecer políticas públicas. Uma política de governo visando o aproveitamento da água pluvial para consumo geraria efeitos positivos na economia, possuindo um caráter distributivo.

Com um projeto preestabelecido, que poderia contemplar a construção de mais cisternas para as residências que não possuem, entrega de kits para instalação de tubulações para captação de água pluvial em residências que já possuem cisternas ou subsídio, o gasto/investimento público com este intuito ajudaria na meta de melhorar o nível de emprego, o que ensejaria a demanda por trabalho (dada à necessidade de trabalhadores para se engajar na execução do projeto), gerando renda (água como indutor do crescimento), resultando no aumento do consumo e consequente crescimento econômico, com possível melhoria distributiva na renda. Destacam-se ainda os gastos que o governo teria disponibilizando os kits, o que estimularia as casas de materiais de construção, impactando o comércio/economia local. Isto significa efeitos sobre três metas de política macroeconômica (aumento de emprego, melhora na distribuição de renda e crescimento), estimulando o desenvolvimento da economia dos municípios do sistema coletivo do sertão.

Importante observar que a região de estudo tem sido objeto constante de investimentos vultosos ligados a alternativas de abastecimento de água, vide a obra do canal adutor do sertão, que segundo Carvalho et al. (2018) ao analisar os Planos Plurianuais de Alagoas verificaram a evolução dos investimentos para o canal contínua e crescente, encontrando-se na ordem de $\mathrm{R} \$ 560$ milhões para Plano Plurianual 2016 - 2019, que pode ser utilizado direto ou indiretamente atrelados ao canal do sertão como planos para o uso da água e projetos de irrigação. Tomando por base a estimativa realizada neste trabalho, verifica-se que um investimento governamental num projeto de aproveitamento de água pluvial como o discorrido neste trabalho representa $1 \%$ do valor previsto para ações ligadas ao canal do sertão alagoano, mostrando-se uma parcela ínfima no contexto de política pública adotada pelo estado, mesmo considerando que essa estimativa inicial pode ainda aumentar devido aos custos dos encargos sociais e administrativos (contratos, licitações e etc.).

\section{Conclusão}

Tendo em vista os aspectos apresentados nesse artigo é possível perceber que a proposta do mesmo é uma solução efetiva e funciona como outra iniciativa para famílias do sertão alagoano terem acesso à água potável. Essa interferência tem grau de promoção social nas localidades, proporcionando um desenvolvimento econômico com o impacto no comércio local e na geração de empregos. $O$ envolvimento das famílias nessa ação favorece um novo significado de coletividade, no qual há cidadãos mais conscientes e atuantes na promoção de novas iniciativas para melhorar o bem-estar da comunidade que está inserido. Sendo assim, o custo mensurado nos resultados para a implantação do sistema terá um retorno futuro muito enaltecedor no campo social, assim com também uma provável redução na tarifa de água das casas beneficiadas pela implantação do sistema de aproveitamento de água pluvial. 


\section{Agradecimentos}

À Universidade Federal de Alagoas - Campus do Sertão por proporcionar a chance de expandir horizontes. À ProEx pelo apoio e incentivo a pesquisa. Aos Professores Thiago Alberto da Silva Pereira e Everton Coelho de Souza, pela orientação e materiais necessários para as pesquisas, mas também pelo apoio, que acompanharam e confiaram na realização deste trabalho. Aos meus colegas que se doaram e se comprometeram na execução deste trabalho.

\section{Referências}

ASSOCIAÇÃO BRASILEIRA DE NORMAS TÉCNICAS. NBR 15527: Água da Chuva Aproveitamento de coberturas em áreas urbanas para fins não potáveis - Requisitos, 2007.

ANA - Agência Nacional de Águas. 2009. Disponibilidade e Demandas de Recursos Hídricos no Brasil. Disponível em <http://www3.ana.gov.br/>. Acesso em 18 março de 2018.

ANDRADE, S. L. P de. "Dimensionamento de Cisternas Tipo Calçadão Associado à Variabilidade Pluviométrica: Estudo de Caso no Município de Delmiro Gouveia - AL", 2015.

Brasil das Águas. A Importância da Água. Disponível em <http://brasildasaguas.com.br/educacional/aimportancia-da-agua/>. Acesso em 18 março de 2018.

CARVALHO, R. S.; DAMASCENO, L. M.; LEVINO, N. A.; PEREIRA, T. A. S. (2018). "Avaliação das Políticas Públicas: Uma Análise das Ações do Canal do Sertão Alagoano" in $70^{a}$ Reunião Anual da SBPC, Maceió, Jul. 2018

CASAL - Companhia de Saneamento de Alagoas. Informações sobre o Sistema Coletivo do Sertão.

GHISI, E.; MONTIBELLER A.; SCHMIDT. R. W.; "Potencial de economia de água potável com uso de água da chuva no setor residencial do Brasil" - Construção e Meio Ambiente, 2006.

IBGE - Instituto Brasileiro de Geografia e Estatística. 2012. Pesquisa Nacional de Amostra por Domicílio e Dados Populacionais. Disponível em <https://www.ibge.gov.br> Acesso em 20 de março de 2018.

LASWELL, H.D. Politics: Who Gets What, When, How. Cleveland, Meridian Books. 1958.

LOWI, Theodor. American Business, Public Policy, Case Studies and Political.

LOWI, Theodor. Four Systems of Policy, Politics, and Choice. Public.

LYNN, L. E. Designing Public Policy: A Casebook on the Role of Policy

PENA, Rodolfo F. Alves. "Escassez de água no Brasil"; Brasil Escola. Disponível em $<$ https://brasilescola.uol.com.br/geografia/escassez-agua-no-brasil.htm>. Acesso em 15 de março de 2018.

SNIS - Sistema Nacional de Informações sobre Saneamento. 2013. Informações sobre o Abastecimento. Disponível em <http://www.snis.gov.br/> Acesso em 20 de março de 2018.

VASCONCELLOS, M. A. S.; GARCIA, M. E. Fundamentos de Economia. 5ª Ed. Saraiva, 2014. 